\title{
APLICAÇÃO DO ESCORE MELD EM PACIENTES SUBMETIDOS A TRANSPLANTE DE FÍGADO: análise retrospectiva da sobrevida e dos fatores preditivos a curto e longo prazo
}

\author{
Ilka de Fátima Santana Ferreira BOIN', Marília Iracema LEONARDI', Elisabete Yoko UD01, \\ Tiago SEVÁ-PEREIRA'1 Raquel Silveira Bello STUCCHI² e Luiz Sergio LEONARDI
}

RESUMO - Racional - Utiliza-se o escore MELD (Model End-Stage Liver Disease) para o prognóstico da mortalidade em lista de espera para transplante de fígado e, em alguns estudos, para predição da sobrevida pós-operatória a longo prazo. Objetivo - Verificar a aplicação do escore MELD como predição da sobrevida após o transplante. Métodos - Por intermédio de dados coletados prospectivamente efetuou-se um estudo de coorte longitudinal retrospectivo em 232 pacientes. Excluíram-se os retransplantes, insuficiência hepática aguda, crianças e enxertos duplos ou reduzidos. Avaliaram-se os dados dos doadores: idade, sexo, peso, creatinina, bilirrubina, sódio, aspartato aminotransferase, antecedentes pessoais, causa da morte, presença de esteatose, número de critérios expandidos do doador e índice de risco do doador. Em relação aos receptores, analisaram-se as variáveis: sexo, idade, peso, doença hepática, pontos de Child-Turcotte-Pugh, escore MELD, depuração de creatinina, sódio, tempos de isquemia e de hospitalização, quantidade de hemoderivados transfundidos, presença e grau de disfunção do enxerto. A análise estatística foi efetuada usando-se a análise de regressão univariada e/ou múltipla, estatística 'c', teste exato de Fisher, método de Kaplan-Meier (teste log-rank) para sobrevida, e análise de regressão de Cox para risco de óbito ajustado para as condições clínicas. Resultados - O ponto de corte MELD para sobrevida foi 20 e de Child-Turcotte-Pugh foi 11,5. Para escore MELD maior ou igual a 20, os fatores preditivos de sobrevida foram: volume de sangue transfundido, disfunção do enxerto e o sódio do doador. Para os hiponatrêmicos os fatores preditivos de sobrevida foram: volume de sangue transfundido, disfunção do enxerto e sódio do doador. A sobrevida estimada para pacientes com escore MELD $\geq 25$ foi menor ao final de 12 meses (68,86\% vs 39,13\%). A sobrevida estimada para os pacientes sem hiponatremia foi maior $(65,16 \%$ vs 44,44\%). A sobrevida aos 5 e 10 anos também seguiu o mesmo padrão. $\mathrm{O}$ uso de doadores limítrofes não alterou a sobrevida, mas quando se utilizou o índice de risco do doador observou-se que a sobrevida foi maior para pacientes com índice de risco do doador menor que 1,7 (63,62\% vs 53,70\%). A associação deste índice com o escore MELD não mostrou diferença estatística em relação à sobrevida. Observou-se que a falência e disfunção do enxerto foram associadas ao número crescente de critérios expandidos do doador. Os receptores de doadores maiores de 50 anos tiveram menor sobrevida (65,58\% vs $38,40 \%)$ e o escore delta-MELD não discriminou a sobrevida. Conclusão - A sobrevida dos receptores a curto e longo prazo é associada a escores MELD acima de 25 , ao volume de sangue transfundido, à disfunção do enxerto, à hiponatremia, à idade do doador acima de 50 anos e àqueles doadores com índice de risco do doador acima de 1,7.

DESCRITORES - Transplante de fígado. Sobrevida. Doadores de tecidos. Índices de gravidade de doenças.

\section{INTRODUÇÃO}

O transplante de fígado vem sendo usado como terapia para doenças hepáticas terminais desde a década de 80, após sua aprovação pelo National Health Institute (EUA), em 1983. Desse tempo em diante, o número de transplantes de fígado no mundo tem aumentado e, conseqüentemente, constatou-se aumento na mortalidade nas listas de espera dos centros transplantadores em geral.

Uma estratégia utilizada, habitualmente, tem sido a utilização de doadores conhecidos como não ideais, limítrofes, com critério expandido ou marginais. Doadores apresentando sorologias para hepatite B ou C, enxertos com esteatose em graus variáveis, doadores depois de

${ }_{1}^{1}$ Unidade de Transplante de Fígado do Hospital de Clínicas da Faculdade de Ciências Médicas da Universidade Estadual de Campinas - FM-UNICAMP; Departamentos de ${ }^{2}$ Clínica Médica e de ${ }^{3}$ Cirurgia da FM-UNICAMP.

Correspondência: Dra. Ilka F. S. Ferreira Boin - Rua Aldo Oliveira Barbosa, 184 - Parque das Universidades - 13086-030 - Campinas, SP. E-mail: ilkaboin@gmail.com 
parada cardíaca e enxertos provenientes de doadores idosos têm sido utilizados com resultados aceitáveis. Outra estratégia utilizada tem sido a aplicação de fígado bipartido e a doação intervivos.

Mesmo com a adoção destes novos critérios, observam-se ainda, mundialmente, altos índices de mortalidade em lista de espera.

Desde 1968 realizam-se transplantes de fígado no Brasil. O programa de captação de órgãos foi iniciado primeiramente em São Paulo, em 1991 e a distribuição era feita para as equipes credenciadas junto à Secretaria de Estado da Saúde do Estado de São Paulo. Após este período, criaram-se as leis sobre a autorização familiar para fins de realização de transplante cadavérico e sobre o cadastramento de candidatos a receptores de fígado com doador cadáver ${ }^{(7,8,9,10,11)}$.

Diversos escores médicos são usados como modelos de prognóstico de gravidade na literatura médica - alguns focalizam a saúde em geral e outros são específicos de algumas doenças. A classificação de Child-Turcotte modificada por PUGH et al. ${ }^{(42)}$, em 1973, foi o modelo vigente até o final da década de 90 para a avaliação da doença hepática crônica. Recentemente, o escore MELD (Model End-Stage Liver Disease), foi descrito por MALINCHOC et al. ${ }^{(34)}$, em 2000, como um modelo matemático capaz de predizer a sobrevida nos primeiros 3 meses em pacientes submetidos a colocação da derivação percutânea transjugular intra-hepática portossistêmica (TIPS).

FREEMAN e EDWARDS ${ }^{(21)}$ conduziram um estudo e reportaram que o tempo em lista de espera não deveria ser o único critério utilizado para alocação de órgãos, pois não estaria relacionado com a mortalidade observada em lista; também referiram que já havia evidência de que a sobrevida após transplante era menor em pacientes mais graves.

Em 2001, KAMATH et al. ${ }^{(30)}$ relataram estudo validando o escore MELD como medida de risco de mortalidade para pacientes com doença hepática crônica em fase terminal e que poderia ser utilizado como ordenação de gravidade para alocação de órgãos. Outras publicações mostraram que a presença de hiponatremia e ascite persistente pode auxiliar a identificação de um tipo de paciente que apresenta alto índice de mortalidade a despeito de escores MELD, usualmente menores que $15^{(28)}$.

Alguns autores, no Brasil, procuraram estudar a utilização desse critério de ordenação de gravidade, seja comparando a classificação de Child-Turcotte-Pugh (CTP) mostrando sua validade ou questionando a alocação de órgãos por lista única pela gravidade do doente com doença hepática crônica ${ }^{(6,14,36,47,48,49,50)}$.

Segundo BAMBHA e KAMATH ${ }^{(3)}$, a introdução do escore MELD tem melhorado a locação de órgãos para transplante de fígado. FREEMAN ${ }^{(22)}$ e FREEMAN et al. ${ }^{(21,23,24)}$ reportaram que houve na era pós-MELD 12\% menos inscrições de pacientes com MELD menores do que 10 . Houve aumento de $10,2 \%$ no número de transplantes realizados e diminuição de 3,5\% de óbito em lista de espera.

O escore MELD foi maior na era pós do que na pré-MELD. Verificaram também que não houve diferenças estatísticas significantes quando se analisou a sobrevida pós-transplante de fígado, embora o paciente mais grave (conceito de "sickest first" (em pior estado clínico)), tivesse sido transplantado e o tempo de lista tenha sido reduzido de 656 para $416 \operatorname{dias}^{(2,3,20,21,31)}$.

Estudo de 105 pacientes com síndrome hepatorrenal sugere que o escore MELD pode ser bom preditor de mortalidade para pacientes com síndrome hepatorrenal tipo 2 em pacientes com MELD maior ou igual a $20^{(1,2)}$. A sobrevida em 3 meses foi maior que $90 \%$ para pacientes com MELD menor ou igual a 20 e de $60 \%$ para os com MELD maior que 20 . O escore MELD mostrouse como melhor preditor de sobrevida do que a falência renal isolada, provavelmente porque o modelo matemático abrange as avaliações hepática e renal concomitantemente.

Em 2006, o consenso nacional (MESSAGE) para estudos do escore $\operatorname{MELD}^{(4,5,6,7,8,9,10,11,12,13,14,15,16,17, ~ 18, ~ 19, ~ 20, ~ 21, ~ 23, ~ 25) ~ n a ̃ o ~}$ incorporou o sódio sérico ou a presença de ascite ao escore MELD.

FENG et al. ${ }^{(19)}$, em estudo realizado em 20.023 transplantes de fígado, analisaram 90.882 doadores (UNOS/OPTN-USA) ${ }^{(18)} \mathrm{em}$ receptores adultos e identificaram fatores com significante risco de perda do enxerto. Por meio do modelo de regressão de Cox conseguiram identificar sete características que, independentemente, foram capazes de predizer riscos de falência de enxerto de modo significativo (idade do doador acima de 40 anos, doação após parada cardíaca, realização de bipartição do fígado, ser afroamericano, altura e causa da morte encefálica). Criaram um índice de risco do doador (IRD) que pode ser informado aos centros transplantadores.

SILBERHUMER et al. ${ }^{(48)}$, em estudo retrospectivo, examinaram uma combinação entre o escore MELD, delta-MELD e doador com critério expandido (DCE). Concluíram que o delta-MELD foi significantemente maior em pacientes que foram a óbito e, quando associado a pelo menos dois critérios expandidos, foi significantemente associado à falência do enxerto e óbito. Concluíram que receptores mais graves não devem receber órgãos de DCE.

Questionamento feito por CHOLONGITAS et al. ${ }^{(15)}$ foi o fato de, estando o paciente mais grave em primeiro lugar em lista de espera, nem sempre receberá o melhor órgão e sim o que lhe for designado e certamente esta atitude não lhe dará a melhor chance de sobrevida. Descrevem ainda que o impacto da mortalidade foi avaliada em 15 estudos envolvendo 20.456 pacientes. Em 11 estudos (19.311 pacientes), observaram níveis de escore MELD entre 24-40, com baixa probabilidade de sobrevida ${ }^{(24)}$.

No Brasil a ordenação do critério MELD foi feita em maio de $2006^{(8)}$, mas a análise do presente estudo foi proposta para os pacientes submetidos a transplante antes da Portaria Ministerial 1160/GM.

O objetivo deste estudo foi verificar a aplicação do escore MELD como predição da sobrevida após o transplante.

\section{MÉTODOS}

A presente investigação foi realizada por intermédio de um banco de dados coletados prospectivamente. Trata-se de estudo de coorte longitudinal retrospectivo, feito em um único centro, com 312 transplantes de fígado realizados em 290 pacientes na Unidade de Transplante Hepático do Hospital de Clínicas da 
Faculdade de Ciências Médicas da UNICAMP, Campinas, SP, no período de junho de 1994 a julho de 2006.

\section{População de estudo}

Os pacientes incluídos, independentemente da raça ou do sexo, eram adultos (maiores de 18 anos) e foram submetidos a transplante de fígado pela técnica de preservação da veia cava do receptor ("piggy-back"), independentemente do tipo de reconstrução venosa supra-hepática realizada.

Dentre os 312 transplantes de fígado excluíram-se os 22 retransplantes realizados. Dos 290 pacientes foram excluídos 58 (crianças, insuficiência hepática fulminante, uso de técnica tradicional, uso de enxertos duplos, dextrocárdicos e uso de enxertos reduzidos).

Os 232 pacientes foram estudados desde a inclusão na lista e realização do transplante, sendo os mesmos acompanhados no Ambulatório da Unidade de Transplante Hepático da instituição até a ocorrência do óbito ou por solicitação de transferência para outro centro transplantador.

Todos receberam o enxerto hepático de doadores cadáveres. A distribuição vigente foi por ordem cronológica de inscrição, segundo as portarias ministeriais vigentes até julho de 2006.

As variáveis coletadas do doador, fornecidas pela Organização de Procura de Órgãos (OPO-UNICAMP), foram: sexo, idade (anos), raça, peso $(\mathrm{kg})$, altura $(\mathrm{cm})$, tipo sangüíneo, tempo de tubagem orotraqueal (horas), valor da creatinina sérica $(\mathrm{mg} / \mathrm{dL})$, valor da bilirrubina total sérica $(\mathrm{mg} / \mathrm{dL})$, valor do sódio sérico $(\mathrm{mEq} / \mathrm{L})$, valor da aspartato aminotransferase (AST) sérica em UI/L, antecedente de alcoolismo (sim ou não), antecedente de infecção na internação que precedeu a morte encefálica ( $\operatorname{sim}$ ou não), antecedente de parada cardiorespiratória $(\mathrm{PCR})$ na internação que precedeu à morte encefálica (sim ou não), antecedente de hipotensão arterial na internação onde ocorreu a morte encefálica (sim ou não), causa da morte encefálica, local de retirada e distância $(\mathrm{km})$ até o centro transplantador, presença de esteatose macro ou microgoticular (a biopsia hepática foi realizada quando a macroscopia indicava alguma alteração) e número de critérios expandidos.

As variáveis pré-operatórias do receptor, anotadas imediatamente antes da realização do transplante, foram: sexo, idade, peso $(\mathrm{kg})$, altura $\left(\mathrm{cm}\right.$ ), IMC (índice de massa corporal em $\mathrm{kg} / \mathrm{m}^{2}$ ), doença, CTP e data da cirurgia. Os valores a seguir foram os da data da inclusão do paciente em lista e aqueles imediatamente anterior à cirurgia do transplante: international normalized ratio (INR) bilirrubina total sérica $(\mathrm{mg} / \mathrm{dL})$, valor da creatinina sérica $(\mathrm{mg} /$ $\mathrm{dL})$, depuração de creatinina estimada $\left(\mathrm{mL} /\right.$ minuto/1,73 $\left.\mathrm{m}^{2}\right)$, sódio sérico $(\mathrm{mEq} / \mathrm{L})$, glicemia $(\mathrm{mg} / \mathrm{dL})$, albumina $(\mathrm{g} / \mathrm{dL})$. Calculou-se o escore MELD na data de inclusão do paciente em lista e imediatamente antes do transplante.

As variáveis intra-operatórias do receptor foram: a) em minutos: tempo de isquemia quente, tempo de isquemia fria, tempo da hepatectomia e tempo de cirurgia; b) em unidades transfundidas: concentrados de hemácia, plasma fresco congelado, plaquetas, albumina, crioprecipitado e sangue recuperado $(\mathrm{mL})$.

As variáveis pós-operatórias do receptor foram: tempo de estadia na UTI (dias), tempo de tubagem orotraqueal (horas), tempo de internação hospitalar (dias), valor máximo da creatinina sérica nos primeiros 7 dias $(\mathrm{mg} / \mathrm{dL})$, depuração de creatinina referente a este valor, valor máximo de alanino aminotransferase (ALT) nos primeiros 7 dias (UI/L), valor máximo de INR nos primeiros 7 dias, realização ou não de retransplante, grau de disfunção do enxerto, presença ou não de falência primária do enxerto, causa da morte e registro da data da última visita até 30 de dezembro de 2006 ou do óbito.

\section{Definição de conceitos}

\section{a) Escore MELD}

O escore MELD foi calculado segundo a fórmula abaixo, disponível em: http://www.unos.org/resources/MELDPeldCalculator.asp

$\left\{0,957 \times \log _{\mathrm{e}}\right.$ [creatinina $\left.(\mathrm{mg} / \mathrm{dL})\right]+0,378 \times \log _{\mathrm{e}}$ [bilirrubina $\left.(\mathrm{mg} / \mathrm{dL})]+1,120 \times \log _{\mathrm{e}}(\mathrm{INR})+0,643\right\} * 10$

Os receptores foram analisados em dois grupos acima ou igual a MELD 25 e acima ou igual a MELD 20 para efeito de análise e correlação com outras variáveis.

\section{b) Delta-MELD}

$\mathrm{O}$ escore delta-MELD ${ }^{(17,21)}$ foi definido como sendo o valor do MELD calculado na data da cirurgia do transplante de fígado subtraído do valor do MELD na época da inscrição do paciente na Central de Transplante da Secretaria do Estado da Saúde de São Paulo.

\section{c) Depuração de creatinina}

A depuração de creatinina foi calculada segundo a fórmula de COCKCROFT e GAULT ${ }^{(16)}=[\{140$-idade $($ anos $)\}$ x peso $(\mathrm{kg}) / 72$ x creatinina sérica $(\mathrm{mg} / \mathrm{dL})]$ x 0,85 (para mulheres), medida esta expressa em $\mathrm{mL} / \mathrm{min} / 1,73 \mathrm{~m}^{2}$.

\section{d) Classificação de Child-Turcotte-Pugh (CTP)}

Utilizou-se a classificação de Child-Turcotte, modificada por Pugh et al. em $1973^{(43)}$, para a colocação dos pacientes em lista única de espera de transplante de fígado(7).

\section{e) Hiponatremia}

Foi definido como hiponatremia a concentração de sódio sérico igual ou inferior a $130 \mathrm{mEq} / \mathrm{L}$, de acordo com a classificação do Clube Internacional de Ascite ${ }^{(32)}$.

\section{f) Graus de disfunção do enxerto}

Foi considerado como PCR o paciente que foi a óbito durante a realização do transplante de fígado, independente da causa. Foi considerado como falência primária do enxerto o paciente que foi a óbito nos primeiros 7 dias, segundo o conceito da UNOS (United Network Organ Sharing $)^{(18)}$, que a define como presença de AST maior que $5000 \mathrm{UI} / \mathrm{L}$ e INR maior ou igual a 2,5 ou acidose (pH $<7,30$ - arterial ou $\mathrm{pH}<7,25$ - venoso) ou lactato maior ou igual a $4 \mathrm{mMol} / \mathrm{L}$ ). Foi considerado como disfunção primária do enxerto o paciente que apresentou disfunção grave do enxerto e que foi a óbito nos primeiros 30 dias. Foi considerado como disfunção do enxerto (DF) o paciente que apresentou dosagem 
TABELA 1. Descrição das variáveis contínuas dos receptores de fígado

\begin{tabular}{|c|c|c|c|c|c|}
\hline Variável & $\mathrm{n}$ & média & mínimo & máximo & DP \\
\hline Pontos (Child-Pugh) & 232 & 9,93 & 5,00 & 15,00 & 2,00 \\
\hline Tempo em lista de espera (meses) & 232 & 16,14 & 0,87 & 61,17 & 11,17 \\
\hline MELD inicial & 228 & 16,89 & 6,00 & 35,00 & 4,68 \\
\hline MELD pré-operatório & 232 & 18,37 & 8,00 & 36,00 & 4,67 \\
\hline Delta-MELD & 228 & 1,43 & $-14,00$ & 19,00 & 4,42 \\
\hline Sódio $(\mathrm{mEq} / \mathrm{L})$ & 232 & 135,06 & 113,00 & 147,00 & 5,38 \\
\hline Depuração de creatinina pré-operatória & 232 & 103,61 & 23,07 & 364,38 & 43,24 \\
\hline Tempo de isquemia quente (minutos) & 229 & 65,74 & 30,00 & 255,00 & 29,58 \\
\hline Tempo de isquemia fria (minutos) & 232 & 710,63 & 330,00 & 1320,00 & 180,03 \\
\hline Tempo de UTI (dias) & 232 & 12,51 & 1,00 & 240,00 & 23,41 \\
\hline Tempo de hospitalização (dias) & 232 & 20,28 & 1,00 & 240,00 & 26,34 \\
\hline Hemácia (unidades) & 232 & 7,08 & 0,00 & 62,00 & 7,64 \\
\hline Recuperação de sangue (mL) & 202 & 1899,23 & 0,00 & 27850,00 & 3317,71 \\
\hline ALT (UI/L) pós-operatória & 223 & 2656,05 & 13,0 & 23280,00 & 3499,01 \\
\hline
\end{tabular}

$\mathrm{ALT}=$ alanina aminotransferase

UTI = unidade de terapia intensiva

$\mathrm{DP}=$ desvio padrão

sérica de ALT acima de 1000 UI/L (DF1); DF2 quando a ALT ficou entre $1001 \mathrm{UI} / \mathrm{L}$ e 2499 e DF3 quando a dosagem foi maior que $2500 \mathrm{UI} / \mathrm{L}$. Esta classificação foi adaptada dos critérios de HEISE et al. ${ }^{(27)}$, em 2003.

\section{g) $D C E$}

DCE ficou como sendo o número de variáveis do doador fora do padrão ideal ${ }^{(12)}$ proposto por SILBERHUMER et al. ${ }^{(48)}$, que variaram de 1 até 5 .

\section{h) IRD}

Utilizou-se o IRD relatado por FENG et al. ${ }^{(19)}$, que leva em consideração o tempo de isquemia fria, a causa da morte do doador (traumática, anóxica, acidente vascular hemorrágico e outras; a idade, altura, distância da cidade de procedência e raça do doador (branca, negra e outras)). Foram agrupados em intervalos de risco, relacionados à probabilidade de sobrevida, conforme citação dos autores, em intervalos: IRD menor ou igual a 1,20; entre 1,21 e 1,40; entre 1,41 e 1,60; entre 1,61 e 1,80 ; maior que 1,81 e o ponto de corte foi 1,7 , de acordo com MALUF et al. ${ }^{(35)}$.

Para estudo da probabilidade de sobrevida, a idade ideal considerada 50 anos, utilizando como referência HOOFNAGLE et al. ${ }^{(29)}$.

A anestesia utilizada foi geral, endovenosa, com ventilação controlada mecânica e monitorização hemodinâmica contínua, de acordo com o protocolo do Departamento de Anestesiologia da Faculdade de Ciências Médicas da UNICAMP.

O protocolo de imunossupressão utilizado foi o esquema tríplice com: a) inibidores de calcineurina: ciclosporina A na dose de 4-8 mg/kg/dia em duas doses diárias, procurando-se alcançar níveis entre $200-400 \mathrm{ng} / \mathrm{mL}$ nos primeiros 3 meses, 150 $300 \mathrm{ng} / \mathrm{mL}$ até o $6^{\circ}$ mês e de $80-150 \mathrm{ng} / \mathrm{mL}$ após o transplante ou tacrolimus na dose de $0,1 \mathrm{mg} / \mathrm{kg} / \mathrm{dia}$, em duas doses diárias, procurando-se alcançar níveis entre $8-12 \mathrm{ng} / \mathrm{mL}$ nos primeiros 6 meses e entre 5-10 ng/mL após; b) inibidores da síntese de purina: azatioprina $50 \mathrm{mg}$ /dia (usada até 1999), micofenolato mofetil na dose de 1000 a $2000 \mathrm{mg}$ /dia após 2000 e micofenolato sódico na dose de 720 a 1480 mg/dia, após 2006; c) corticoesteróides: metilprednisolona $1,0 \mathrm{~g}$ após a reperfusão, $200 \mathrm{mg}$ no $1^{\circ}$ dia, $100 \mathrm{mg}$ no segundo e redução até $20 \mathrm{mg}$ até o $7^{\circ}$ dia e manutenção desta dose até o $30^{\circ}$ dia, $15 \mathrm{mg}$ até o $60^{\circ}$ dia, $10 \mathrm{mg}$ até o $90^{\circ}$ dia pós-operatório e retirada do medicamento, com exceção dos pacientes com doença auto-imune.

\section{Análise estatística}

A caracterização das variáveis dos casos da amostra encontrase na Tabela 1. A curva ROC ("receiver operating characteristic curve") foi utilizada para se verificar o poder de discriminação do escore MELD, delta-MELD, e CTP para a predição de mortalidade. Para se verificar associação ou comparar proporções foi utilizado o teste do Qui ao quadrado ou teste exato de Fisher, quando necessário.

O estudo dos vários fatores associados ao óbito foi realizado por meio da análise de regressão logística (modelo logito). Inicialmente, foi realizada análise univariada de cada um dos fatores de interesse, previamente selecionados por sua importância clínica e a seguir foi feita análise múltipla, usando-se o critério passo a passo.

A análise da sobrevida foi realizada utilizando-se o método de Kaplan-Meier e a comparação entre as distribuições foi realizada usando-se o teste log-rank. Utilizou-se a análise de regressão de Cox para a variável tempo de sobrevida ajustada para as condições clínicas. O nível de significância $(P)$ adotado em todas as análises foi de 5\%. Foi utilizado o programa Statistica 7.0 (2005) - EUA para a realização dos testes estatísticos e gráficos.

\section{RESULTADOS}

Dos 232 pacientes, $170(73,3 \%)$ eram homens e $62(26,7 \%)$ mulheres, com idade média de $46,4 \pm 10,3$ anos. A hepatite por vírus $\mathrm{C}$ foi a causa mais freqüente $(119=51,1 \%)$. A 
maioria $(83,6 \%)$ dos pacientes apresentou IMC $<30$. A causa da morte encefálica do doador foi traumática em $123(53,1 \%)$, seguida do acidente vascular cerebral hemorrágico em 84 $(36,2 \%)$. Detectou-se que $13(6,9 \%)$ pacientes necessitaram de retransplante.

A utilização da curva ROC mostrou que as variáveis MELD e delta-MELD não foram boas para discriminar significantemente os que sobreviveram, daqueles que foram a óbito no período pós-operatório. O que se pode observar é que o ponto de corte (índice de Youden) foi 20 para MELD, $\geq-1,5$ para delta-MELD, significando que quanto maior o valor, maior é o risco de óbito. Apenas o escore Child-Pugh (corte $\geq 11,5$ ) foi capaz de diferenciar significantemente um grupo do outro $(P=0,02)$.

A influência do número de critérios expandidos do doador sobre a presença de disfunção ou falência do enxerto encontrase na Figura 1.

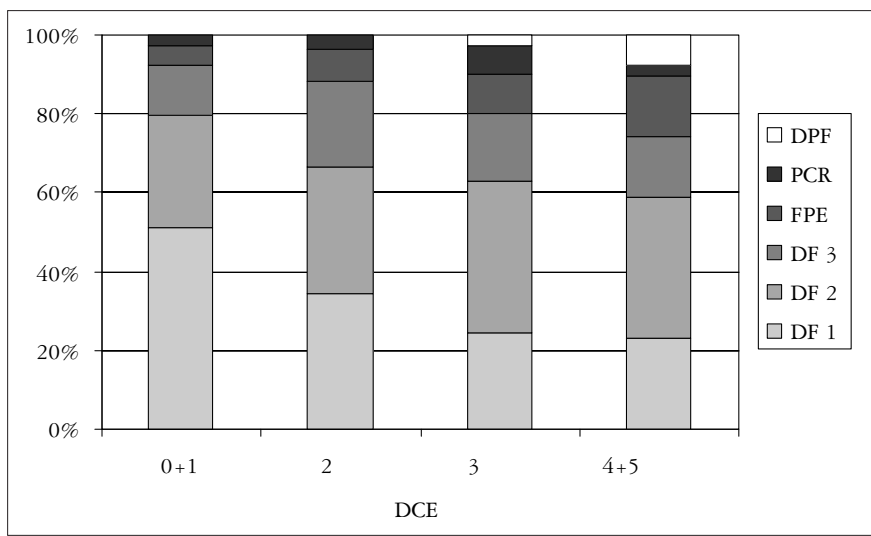

FIGURA 1. Distribuição gráfica dos graus de disfunção do enxerto do receptor de fígado em relação ao número de critérios expandidos (DCE) observados nos doadores, (teste exato de Fisher $=23368, P<0,0001)$; (DF $1=$ pacientes com alanina aminotransferase $<1000 \mathrm{UI} / \mathrm{L} ;$ DF 2 entre 1501 e $2500 \mathrm{UI} / \mathrm{L} ; \mathrm{DF} 3$ = pacientes com alanina aminotransferase $>2500 \mathrm{UI} / \mathrm{L}$ ) sendo que falência primária do enxerto é falência primária do enxerto; DPF é disfunção primária do enxerto, PCR são os pacientes que foram a óbito durante o transplante e DCE é o número de critérios expandidos do doador limítrofe

Após análise de regressão univariada, verificou-se que os pacientes com maior risco de óbito foram: os que receberam maior quantidade de hemácias (a cada unidade de hemácias o risco de óbito aumenta $6 \%$ ou 1,06 vezes), menor valor de depuração de creatinina (a cada unidade, o risco de óbito diminui $2 \%$ ), maior valor de ALT após o transplante (a cada $1.000 \mathrm{UI} / \mathrm{L}$ de ALT o risco de óbito aumenta 24\%) e maior idade do doador (receptores de doadores com 50 ou mais anos de idade têm risco 5 vezes maior de óbito).

A seguir, efetuou-se um modelo múltiplo com as variáveis de hemoderivados e, pelos resultados, verificou-se influência significativa apenas da variável quantidade de hemácias transfundidas em relação ao óbito $(P=0,001 ; \mathrm{OR}=1,14$; IC $=1,06-1,22$ ).
Pela análise da regressão de Cox para a variável dependente 'tempo de sobrevida', estratificada para variável sódio menor ou igual a $130 \mathrm{mEq} / \mathrm{L}$, pode-se observar que para cada unidade a mais de hemácias transfundida a razão de risco aumenta $4 \%$. Para cada $1.000 \mathrm{~mL}$ de sangue recuperado o risco aumenta $8,5 \%$. Para cada $1.000 \mathrm{UI} / \mathrm{L}$ de ALT o risco aumenta $8 \%$.

Pela análise de regressão de Cox para a variável dependente 'tempo de sobrevida', estratificada para a variável MELD acima ou abaixo de 20, pôde-se observar que para cada unidade a mais de hemácias transfundida a razão de risco aumenta 5,2\%. Para cada $1000 \mathrm{~mL}$ de sangue recuperado o risco aumenta $9,5 \%$. Para cada $1.000 \mathrm{UI} / \mathrm{L}$ de ALT o risco de óbito aumenta $5,4 \%$ e para cada unidade a mais de sódio do doador o risco aumenta $2,1 \%$. $\left(\chi^{2}=99,659 ; P<0,0001\right)$.

Observou-se que apresentaram menor sobrevida: os pacientes com MELD igual ou maior que 25 , os pacientes hiponatrêmicos, os receptores com IRD estratificado maior de 1,7 e aqueles que utilizaram doadores acima de 50 anos (Figuras 2 a 5).

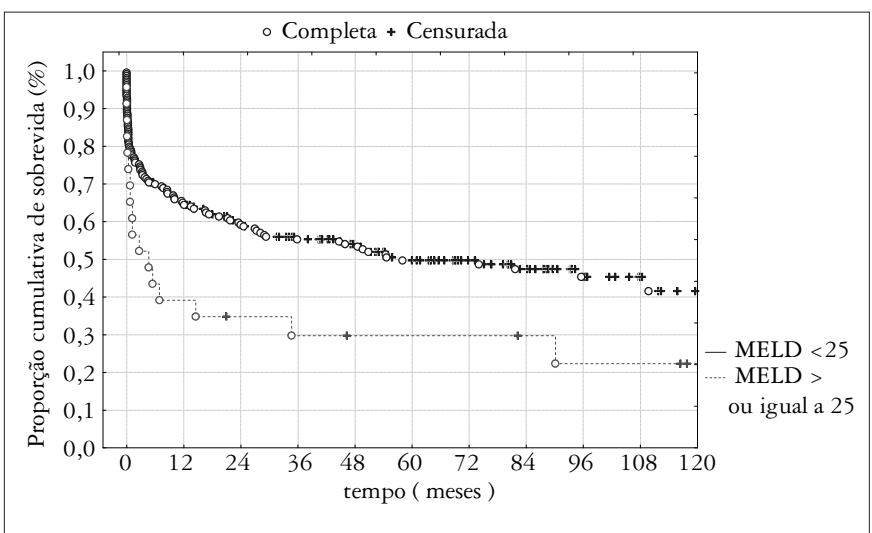

FIGURA 2. Curvas de sobrevida (Kaplan-Meier) dos receptores de fígado com MELD > ou igual a 25 e abaixo de 25 (teste log-rank, $P=0,02$ )

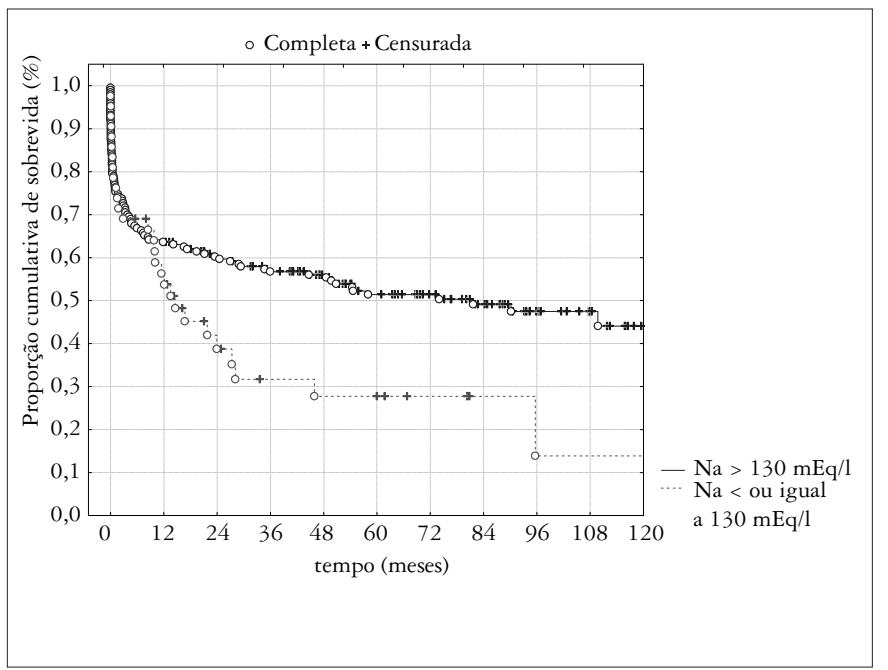

FIGURA 3. Curva de sobrevida dos receptores de fígado de acordo com a dosagem de sódio sérico $(\mathrm{Na})$. A sobrevida foi maior $(P<0,05)$ nos pacientes sem hiponatremia 


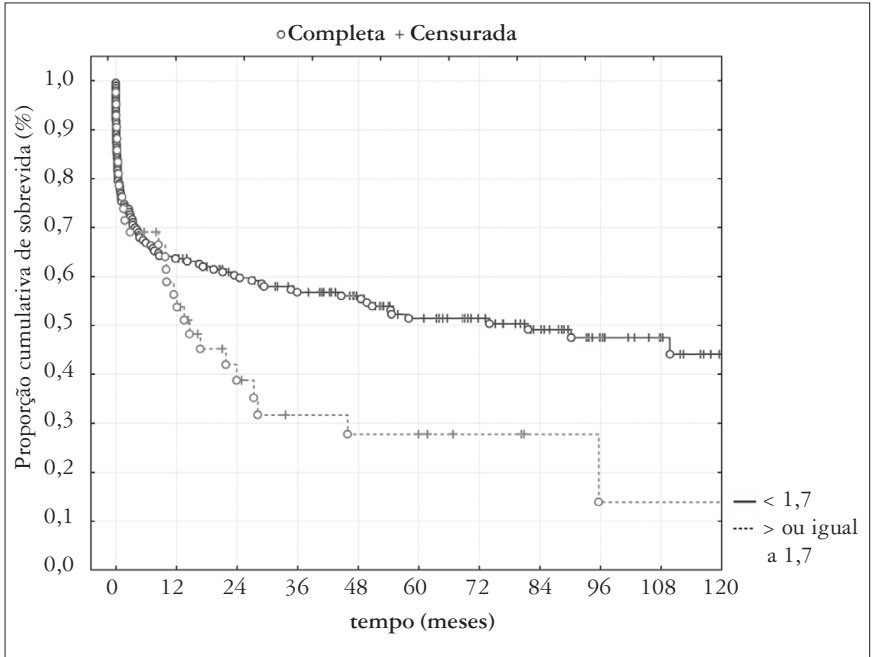

FIGURA 4. Curva de sobrevida utilizando índice de risco de doador (IRD) $>$ ou igual a 1,7 . Nota-se que a sobrevida foi maior nos receptores cujos doadores apresentaram IRD menor que $1,7(P=0,03)$

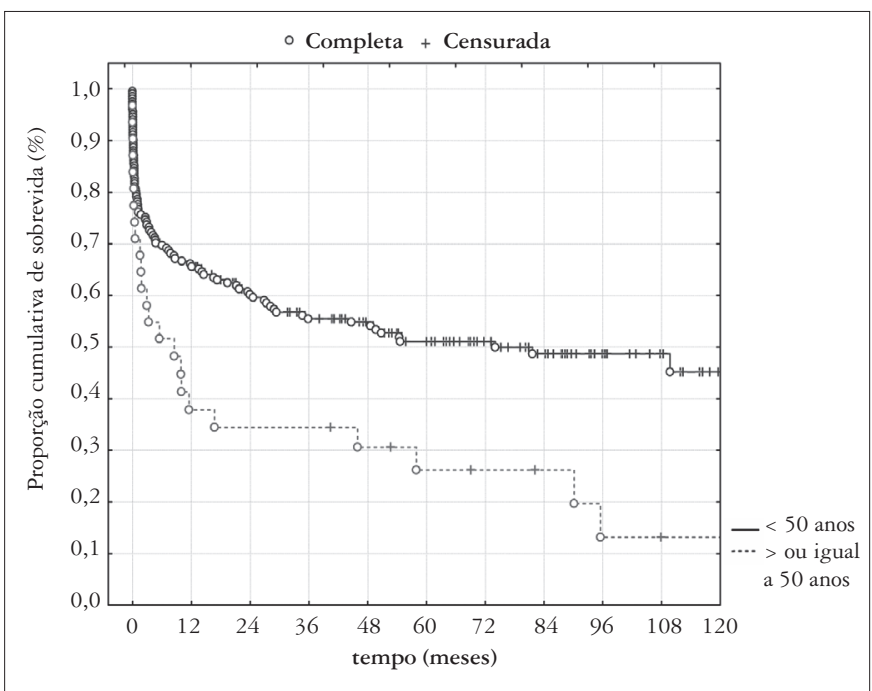

FIGURA 5. Curva de sobrevida dos receptores de fígado utilizando doadores com menos de 50 anos. Houve melhor sobrevida quando se utilizou doadores com menos de 50 anos $(P=0,004)$

\section{DISCUSSÃO}

Os resultados obtidos neste estudo mostraram que a causa mais freqüente das doenças hepáticas que levaram ao transplante foi a hepatite por vírus C. A idade média observada foi de 46,4 anos, com predominância do sexo masculino $(73,28 \%)$. A mortalidade ocorreu mais entre as mulheres. MACHICAO et al. ${ }^{(33)}$ admitem também que a incidência mais freqüente foi de pacientes com hepatite $\mathrm{C}$, do sexo masculino e com idade média semelhante entre os pacientes submetidos a transplante de fígado.
FENG et al. ${ }^{(19)}$ relataram um estudo realizado em 20.023 transplantes de fígado e analisaram 90.882 doadores (UNOS/ OPTN-USA) ${ }^{(18,40)} \mathrm{em}$ receptores adultos e identificaram fatores com significante risco de perda do enxerto. Criaram IRD, que pode ser informado aos centros transplantadores e possibilitar melhores alocações minimizando a morbidade e a mortalidade dos receptores.

Neste estudo, a maior causa de mortalidade do doador foi o trauma cranioencefálico $(53,03 \%)$, seguido de acidente vascular cerebral hemorrágico $(36,20 \%)$. A cidade de procedência do doador variou e observou-se que a captação local foi baixa, devendo ser estimulada tanto a notificação, quanto a busca ativa para que estes índices possam ser melhorados. Quando se procede à captação em cidades mais longínquas, obtém-se, proporcionalmente, maior tempo de isquemia fria. Neste estudo o uso de doadores com IRD maior que 1,7 resultou em menor sobrevida. Entretanto, não foi observada diferença quando este índice foi associado à estratificação do escore MELD.

Na presente série, o uso de doadores com 50 anos ou mais aumentou o risco de óbito em 5 vezes na análise univariada. Mesmo sabendo do risco do uso de doadores 'idosos', observouse na literatura o uso dos mesmos e afirmação indubitável é que, o fundamental para alocação de órgãos em transplante, permanece sendo a necessidade de doadores para que os mesmos ocorram.

Em alguns estudos observa-se que a sobrevida declina rapidamente durante o $1^{\circ}$ ano após o transplante e se mantém em um patamar após este período ${ }^{(26)}$, sendo de $83 \%$ ao final de 12 meses, de $72 \%$ após o $5^{\circ}$ ano e $58 \%$ ao final de 10 anos e a sobrevida para os pacientes com escore MELD acima de 36, ao final do $10^{\circ}$ ano, foi de $40 \%^{(22,23,24,25,26,27)}$. No Brasil a sobrevida tem sido menor e isto pode ser devido ao expressivo número de doadores com critérios expandidos utilizados ${ }^{(45)}$.

No presente estudo, o escore MELD médio e a pontuação de Child-Pugh, e o tempo de isquemia fria foram semelhantes aos encontrados na literatura ${ }^{(26,44)}$, sendo observado que os pacientes de MELD menor que 25 apresentaram maior tempo de sobrevida.

RUSSO $^{(46)}$ ressaltou, analisando alguns trabalhos ${ }^{(4,5,28)}$, que o sódio sérico, independentemente, foi fator de risco para mortalidade em análise multivariada. Em relação à hiponatremia, observou que os pacientes hiponatrêmicos apresentam menor sobrevida a curto e a longo prazo.

MACHICAO et al. ${ }^{(33)}$ afirmaram que a implementação do escore MELD não teve influência significante para se verificar a ocorrência de falência renal (depuração de creatinina abaixo de $60 \mathrm{~mL} / \mathrm{min} / 1,73 \mathrm{~m}^{2}$ ) nos primeiros 2 anos após transplante de fígado, mesmo utilizando receptores com níveis de creatinina mais altos do que na era pré-MELD.

Neste estudo, utilizando os critérios propostos por PARAMESH et al. ${ }^{(41)}$, verificou-se não haver diferença estatisticamente significante em relação à sobrevida, entre pacientes com ou sem disfunção renal.

A prevenção de perda excessiva de sangue é um importante objetivo durante a realização de transplante de fígado, em conseqüência da associação com prolongada recuperação, aumento 
da morbidade e diminuição da sobrevida. Embora transfusões maciças ainda aconteçam, $10 \%$ a $50 \%$ das cirurgias de transplante cursam sem requerer transfusões de sangue ${ }^{(13,37,38,39,43)}$. No presente estudo, $8,62 \%$ dos pacientes não foram submetidos a transfusão sangüínea durante a realização do transplante de fígado.

A transfusão de mais de seis unidades de concentrados de hemácias tem sido relacionada à reduzida sobrevida após o transplante de fígado ${ }^{(43)}$. É notória a associação de politransfusão e complicação pós-operatória, mas poucos estudos têm demonstrado a associação de número de concentrados de hemácias transfundidas como fator preditivo de sobrevida.

XIA et al. ${ }^{(51)}$ estudaram retrospectivamente um grupo de 124 pacientes submetidos a transplante e concluíram que aqueles com escores MELD altos exigiram maior quantidade de hemoderivados e vasopressores. A média do escore MELD obtido pelos autores foi de 22 (no grupo que foi politransfundido) e de 13 (no grupo sem politransfusão). As morbidades associadas à politransfusão foram a presença de síndrome hepatorrenal, de ascite, de encefalopatia e de varizes esôfago-gástricas.

Nesta série observou-se que os pacientes submetidos a politransfusão apresentaram menor sobrevida, incluindo aqueles que utilizaram a recuperação sangüínea autóloga. É interessante esta observação, já que com a aplicação do escore MELD trabalha-se com doentes cada vez mais graves e sujeitos a esta intercorrências clínicas.

O uso do critério MELD é interessante na alocação de órgãos num sistema que envolve grande número de equipes transplantadoras e número significativo de pacientes em lista de espera. Observa-se que os pacientes hiponatrêmicos, não sendo contemplados com situação especial, terão menor sobrevida tanto em lista de espera, conforme relatado na literatura, assim como após a realização do transplante.

A utilização de receptores mais graves associada a doadores com critério expandido, podem levar a menor sobrevida e talvez a utilização do IRD seja útil para melhorar a alocação dos órgãos disponíveis.

Concluindo, observou-se que, embora se tenha estudado e analisado vários parâmetros dos doadores e dos receptores, quanto mais grave o receptor, maiores chances de morbidade e mortalidade deverão ser previstas e coibidas. No Brasil, além da escassez de doadores, pode-se utilizar número substancial de doadores com critérios expandidos. A adição desses critérios tem aumentado o potencial de doação de órgãos, mas torna necessário ajuste entre a oferta de doadores com muitos critérios expandidos a receptores mais graves. A transfusão maciça deve ser evitada, o uso de doadores idosos deve ser criterioso e os pacientes hiponatrêmicos devem ser contemplados com algum tipo de bonificação.

Boin IFSF, Leonardi MI, Udo EY, Sevá-Pereira T, Stucchi RSB, Leonardi LS. The application of MELD score in patients submitted to liver transplantation - A retrospective analysis of survival and the predictive factors in the short and long term. Arq Gastroenterol. 2008;45(4):275-83.

ABSTRACT - Background - The model for end-stage liver disease (MELD) was developed to predict short-term mortality in patients with cirrhosis. There are few reports studying the correlation between MELD and long-term posttransplantation survival. Aim - To assess the value of pretransplant MELD in the prediction of posttransplant survival. Methods - The adult patients (age $>18$ years) who underwent liver transplantation were examined in a retrospective longitudinal cohort of patients, through the prospective data base. We excluded acute liver failure, retransplantation and reduced or split-livers. The liver donors were evaluated according to: age, sex, weight, creatinine, bilirubin, sodium, aspartate aminotransferase, personal antecedents, brain death cause, steatosis, expanded criteria donor number and index donor risk. The recipients' data were: sex, age, weight, chronic hepatic disease, Child-Turcotte-Pugh points, pretransplant and initial MELD score, pretransplant creatinine clearance, sodium, cold and warm ischemia times, hospital length of stay, blood requirements, and alanine aminotransferase (ALT $>1,000 \mathrm{UI} / \mathrm{L}=$ liver dysfunction). The Kaplan-Meier method with the log-rank test was used for the univariable analyses of posttransplant patient survival. For the multivariable analyses the Cox proportional hazard regression method with the stepwise procedure was used with stratifying sodium and MELD as variables. ROC curve was used to define area under the curve for MELD and Child-Turcotte-Pugh. Results - A total of 232 patients with 10 years follow up were available. The MELD cutoff was 20 and Child-Turcotte-Pugh cutoff was 11.5. For MELD score $\geq 20$, the risk factors for death were: red cell requirements, liver dysfunction and donor's sodium. For the patients with hyponatremia the risk factors were: negative delta-MELD score, red cell requirements, liver dysfunction and donor's sodium. The regression univariated analyses came up with the following risk factors for death: score MELD $\geq 25$, blood requirements, recipient creatinine clearance pretransplant and age donor $\geq 50$. After stepwise analyses, only red cell requirement was predictive. Patients with MELD score $<25$ had a $68.86 \%, 50,44 \%$ and $41,50 \%$ chance for 1,5 and 10 -year survival and $\geq 25$ were $39.13 \%, 29.81 \%$ and $22.36 \%$ respectively. Patients without hyponatremia were $65.16 \%, 50.28 \%$ and $41,98 \%$ and with hyponatremia $44.44 \%, 34.28 \%$ and $28.57 \%$ respectively. Patients with IDR $\geq 1.7$ showed $53.7 \%, 27.71 \%$ and $13.85 \%$ and index donor risk $<1.7$ was $63.62 \%, 51.4 \%$ and $44.08 \%$, respectively. Age donor $\geq 50$ years showed $38.4 \%, 26.21 \%$ and $13.1 \%$ and age donor $<50$ years showed $65.58 \%, 26.21 \%$ and $13.1 \%$. Association with delta-MELD score did not show any significant difference. Expanded criteria donors were associated with primary non-function and severe liver dysfunction. Predictive factors for death were blood requirements, hyponatremia, liver dysfunction and donor's sodium. Conclusion - In conclusion MELD over 25, recipient's hyponatremia, blood requirements, donor's sodium were associated with poor survival.

HEADINGS - Liver transplantation. Survivorship (Public health). Tissue donors. Severity of illness index. 


\section{REFERÊNCIAS}

1. Alessandria C, Ozdogan O, Guevara M, Restuccia T, Jimenez W, Arroyo V, Rodés J, Ginès P. MELD score and clinical type predict prognosis in hepatorenal syndrome: relevance to liver transplantation. Hepatology. 2005;41:1282-9.

2. Bajaj JS, Saeian K. MELD score does not discriminate against patients with hepatic encephalopathy. Dig Dis Sci. 2005;50:753-6.

3. Bambha K, Kamath PS. Model for end-stage liver disease (MELD); [acesso em 9 de Janeiro de 2007]. Disponível em: http://www.utdol.com/utd/content/topic. do?topicKey=cirrhosis [WEB005-200.68.81.91-6284CA06C2-6].

4. Biggins SW, Colquhoun S, Gish RG, Runyon BA. Model for end-stage liver disease (MELD). Exceptions for ascites. Liver Transpl. 2006;12:s88-s90.

5. Boin IF, Leonardi MI, Pinto AO, Leme RS, Udo E, Stucchi RS, Soares EC, Leonard LS. Liver transplant recipients mortality on the waiting list: long-term comparison to Child-Pugh classification and MELD. Transplant Proc. 2004;36:920-2

6. Boin I, Leonardi MI, Sevá-Pereira T, Soares EC, Stucchi R, Udo EY, Cardoso AR, Caruy CA, Leonardi LS. Serum sodium receptor before liver transplantation and actuarial survival [resumo]. Liver Transpl. 2006;12(5):LB-8, C117. [Apresentado ao The 2006 Joint International Congress of ILTS, ELTA \& LICAGE; 2006 maio 3-6; Milão-Itália].

7. Brasil. Leis, etc. Decreto n. 2.268 de 30 de junho de 1997. Ministério da Saúde. Dispõe sobre a remoção de órgãos, tecidos e partes do corpo humano para fins de transplante e tratamento e criação do sistema nacional de transplante. Diário Oficial da União, Brasília (DF). 19971 jul. p. 13.739.

8. Brasil. Presidência da República. Ministério da Saúde. Portaria n. 3.407 de 06 de agosto de 1998. Seleção de pacientes para distribuição de fígado-doador cadáver.

9. Brasil. Leis, etc. Lei 10.211 de 23 de março de 2001. Ministério da Saúde.

10. Brasil. Presidência da República. Ministério da Saúde. Portaria n. 541 de 14 março de 2002. Critérios para cadastramento de candidatos a receptores de fígado - doador cadáver. Diário Oficial da União, Brasília (DF). 200214 mar.

11. Brasil. Presidência da República. Ministério da Saúde. Portaria n. 1.160 de 29 de maio de 2006. Critérios para distribuição de fígado doador cadáver para transplante. Diário Oficial da União, Brasília (DF) 200631 maio n.103.

12. Briceño J, Solórzano G, Pera-Madrazo C. A proposal for marginal liver grafts. Transpl Int. 2000;13:s249-s52.

13. Cacciarelli TV, Keefe EB, Moore DH, Burns W, Chuljian P, Busque S, Concepcion W, So EK, Esquivel CO. Primary liver transplantation without transfusion of red blood cells. Surgery. 1996;120:698-704.

14. Certa G, Bertin T, Martines D, Salvagnini M. MELD (model for end-stage liver disease) is more accurate than maddrey discriminant function in order to predict survival of patients with alcoholic hepatitis (AH). Dig Liver Dis. 2002;34:A52.

15. Cholongitas E, Senzolo M, Patch D, Kwong K, Nikolopoulou V, Leandro G, Shaw $\mathrm{S}$, Burroughs AK. Risk factors, sequential organ failure assessment and model for end-stage liver disease scores for predicting short term mortality in cirrhotic patients admitted to intensive care unit. Aliment Pharmacol Ther. 2006;23:883-93.

16. Cockcroft DW, Gault MH. Prediction of creatinine clearance from serum creatinine. Nephron. 1976;16:31-41.

17. D'Amico G. Developing concepts on MELD: delta and cutoffs. J Hepatol. 2005; 42:790-2

18. Estados Unidos. United Network Organ Sharing. [acesso em 15 de abril de 2007] Disponível em: www.unos.org/policiesbylaws/policies e www.unos.org/resources/ MELDPeldCalculator.asp

19. Feng S, Goodrich NP, Bragg-Gresham JL, Dykstra DM, Punch JD, DebRoy MA, Greenstein SM, Merion RM. Characteristics associated with liver graft failure: the concept of a donor risk index. Am J Transplant. 2006;6:783-90.

20. Fink MA, Angus PW, Gow PJ, Berry SR, Wang BZ, Muralidharan V, Christophi C, Jones RM. Liver transplant recipient selection: MELD vs. clinical judgment. Liver Transpl. 2005;11:621-6.

21. Freeman RB Jr, Edwards E. United Network for Organ Sharing Liver and Intestine Committee. Liver transplant waiting time does not correlate with waiting list mortality: implications for liver allocation policy. Liver Transpl. 2000;6:543-52.

22. Freeman RB. Mathematical models and behavior: assessing delta MELD for liver allocation. Am J Transplant. 2004;4:1735-6.

23. Freeman RB, Wiesner RH, Edwards E, Harper A, Merion R, Wolfe R. United Network for Organ Sharing Organ Procurement and Transplantation Network Liver and Transplantation Committee. Results of the first year of the new liver allocation plan. Liver Transpl. 2004;10:7-15.

24. Freeman RB, Harper A, Edwards EB. Excellent liver transplant survival rates under the MELD/PELD system. Transplant Proc. 2005;37:585-8.

25. Freeman RB Jr, Gish R, Harper A, Davis GL, Vierling J, Lieblein L, Klintmalm G, Blazek J, Hunter R, Punch J. Model for end-stage liver disease (MELD) exception guidelines: results and recommendations from the MELD exception study group and conference (MESSAGE) fro the approval of patients who need liver transplantation with diseases not considered by the standard MELD formula. Liver Transpl. 2006;12(Suppl 3):s128-s36.

26. Habib S, Berk B, Chang CC, Demetris AJ, Fontes P, Dvorchik I, Eghtesad B, Marcos A, Shakil O. MELD and prediction of post-liver transplantation survival. Liver Transpl. 2006; 12:440-7.

27. Heise M, Settmacher U, Pfitzmann R, Wunscher, Muller AR, Jonas S, Neuhaus P. A survival-based scoring-system for initial graft function following orthotopic liver transplantation. Transpl Int. 2003;16:794-800.

28. Heuman DM, Abou-Assi SG, Habib A, Williams LM, Stravitz RT, Sanyal AJ, Fisher RA, Mihas AA. Persistent ascites and low serum sodium identify patients with cirrhosis and low MELD scores who are at high risk for early death. Hepatology. 2004; $40: 802-10$.

29. Hoofnagle JH, Lombardero M, Zetterman RK, Lake J, Porayko M, Everhart J, Belle SH, Detre KM. Donor age and outcome of liver transplantation. Hepatology. 1996;24:89-96

30. Kamath PS, Wiesner RH, Malinchoc M, Kremers W, Therneau TM, Kosberg CL D'Amico, Dickson ER, Kim WR. A model to predict survival in patients with endstage liver disease. Hepatology. 2001;33:464-70.

31. Kanwal F, Dulai GS, Spiegel BM, Yee HF, Gralnek IM. A comparison of liver transplantation outcomes in the pre- vs. post-MELD eras. Aliment Pharmacol Ther. $2005 ; 21: 169-77$.

32. Londono MC, Guevara M, Rimola A, Navasa M, Taura P, Mas A, Garcia-Valdecasas JC, Arroyo V, Ginès P. Hyponatremia impairs early posttransplantation outcome in patients with cirrhosis undergoing liver transplantation. Gastroenterology. 2006; 130:1135-43

33. Machicao VI, Srinivas TR, Hemming AW, Soldevila-Pico C, Firpi RJ, Reed AI, Morelli GJ, Nelson DR, Abdelmalek MF. Impact of implementation of the MELD scoring system on the prevalence and incidence of chronic renal disease following liver transplantation. Liver Transpl. 2006;12:754-61.

34. Malinchoc M, Kamath PS, Gordon FD, Peine CJ, RanK J, ter Borg PC. A model to predict survival in patients undergoing transjugular intrahepatic portosystemic shunts. Hepatology. 2000;31:864-71.

35. Maluf DG, Edwards E, Kauffman. Utilization of extended donor criteria liver allograft: is the elevated risk of failure independent of the model for end-stage liver disease score of the recipient? Transplantation. 2006;82:1653-7.

36. Massarollo PCB, Fernandes JH, Millan LS, Inácio CAF, Rodrigues AJ Jr, Mies S Efeito da escala MELD na mortalidade após o transplante de fígado. J Bras Transpl. $2003 ; 6: 14-22$.

37. Massicotte L, Sassine MP, Lenis S, Seal RF, Roy A. Survival rate changes with transfusion of blood products during liver transplantations. Can J Anaesth. 2005;52:148-55.

38. McCluskey AS, Karkouti K, Wijeysundera DN, Kakizawa K, Ghannam M, Hamdy A, Grant D, Levy G. Derivation of a risk index for the prediction of massive blood transfusion in liver transplantation. Liver Transpl. 2006;12:1584-93.

39. Mor E, Jennings L, Gonwa TA, Holman MJ, Gibbs J, Solomon H. The impact of operative bleeding on outcome in transplantation of the liver. Surg Gynecol Obstet. $1993 ; 176: 219-27$

40. Organ Procurement and Transplantation Network - HRSA. Final Rule with commen period. Fed Regist. 1998;63:16296-338 [acesso em 1 de maio de 2007]. Disponível em: www.optn.org.

41. Paramesh AS, Roayaie S, Doan Y, Schwatz ME, Emre S, Fishbein T, Florman S, Gondolesi GE, Krieger N, Ames S, Bromberg JS, Akalin E. Post-liver transplant acute renal failure: factors predicting development of end-stage renal disease. Clin Transpl. 2004;18:94-9.

42. Pugh RNH, Muray Lyon IM, Dawson JL, Pietroni MC, Williams R. Transaction of the oesophagus for bleeding oesophageal varices. Br J Surg. 1973;60:646-9.

43. Ramos E, Dalmau A, Sabate A, Lama C, Lladó L, Figueras J, Jaurrieta E. Intraoperative red blood cell transfusion in liver transfusion: influence on patient outcome, prediction of requirements, and measures to reduce them. Liver Transpl. 2003;9:1320-7.

44. Ravaioli M, Grazi GL, Ercolani G, Cescon M, Varotti G, Del Gaudio M, Vetrone G, Lauro A, Ballardini G, Pinna AD. Efficacy of MELD score in predicting survival after liver retransplantation. Transplant Proc. 2004;36:2748-9.

45. Rocha MB, Boin IFSF, Escanhoela CAF, Leonardi LS. Can the use of marginal live donors change recipient survival rate? Transplant Proc. 2004;36:914-5.

46. Russo MW. MELD and serum sodium. Hepatology. 2005;41:1198-9.

47. Sette H Jr, Bacchella T, Machado MC. Critical analysis of the allocation policy for liver transplantation in Brazil. Rev Hosp Clin Fac Med Sao Paulo. 2003;58:179-84.

48. Silberhumer GR, Pokorny H, Hetz H, Herkner H, Rasoul-Rockenschaub S, Soliman T, Wekerle T, Berlakovich GA, Steininger R, Muehlbacher F. Combination of extended donor criteria and changes in the model for end-stage liver disease score predict patien survival and primary dysfunction in liver transplantation: a retrospective analysis. Transplantation. 2007;83:588-92. 
Boin IFSF, Leonardi MI, Udo EY, Sevá-Pereira T, Stucchi RSB, Leonardi LS. Aplicação do escore MELD em pacientes submetidos a transplante de fígado - análise retrospectiva da sobrevida e dos fatores preditivos a curto e longo prazo

49. Teixeira AC, Souza FF, Mota GA, Martinelli ALC, Sankarankutty AK, Castro e Silva O. Liver transplantation. Expectation with MELD score for liver allocation in Brazil. Acta Cir Bras. 2006;21:12-4

50. Teixeira AC, Souza FF, Sankarankutty AK, Martinelli ALC, Castro e Silva O Jr. Characteristics of waitlisted patients for liver transplantation at a liver transplantation unit in the city of Ribeirão Preto, São Paulo, Brazil, especially considering child and MELD for end stage liver disease (MELD) scores. Transplant Proc. 2007;39:387-9.
51. Xia VW, Du B, Braunfeld M, Neelakanta G, Hu KQ, Nourmand H, Levin P, Enriquez R, Hiatt JR, Ghobrial RM, Farmer DG, Busuttil RW, Steadman RH. Preoperative characteristics and intraoperative transfusion and vasopressor requirements in patients with low vs. high MELD scores. Liver Transpl. 2006;12:614-20.

Recebido em 13/9/2007. Aprovado em 13/6/2008 